\title{
Avaliação da segurança clínica de um fitoterápico contendo Mikania glomerata, Grindelia robusta, Copaifera officinalis, Myroxylon toluifera, Nasturtium officinale, própolis e mel em voluntários saudáveis
}

\author{
Aline K.A. Soares*, Gardênia C. Carmo, Diana P. Quental, Demétrius F. Nascimento, \\ Fernando A.F. Bezerra, Manoel O. Moraes, Maria E.A. Moraes
}

Unidade de Farmacologia Clínica (UNIFAC), Departamento de Fisiologia e Farmacologia, Universidade Federal do Ceará, Rua Cel. Nunes de Melo 1127, Rodolfo Teófilo,

60431-970, Fortaleza, Ce, Brasil

\begin{abstract}
RESUMO: O fitoterápico Calmatoss ${ }^{\circledR}$ é um xarope composto por tinturas de guaco (Mikania glomerata), grindélia (Grindelia robusta), copaíba (Copaifera officinalis), bálsamo de Tolú (Myroxylon toluifera), álcoolatura de agrião (Nasturtium officinale), própolis e mel. Sua principal indicação é para o tratamento de afecções do trato respiratório por suas ações antimicrobianas, antitussígenas, expectorantes e broncodilatadora. O objetivo desse estudo foi avaliar a toxicologia clínica dessa preparação em voluntários saudáveis. Para isso realizou-se um ensaio clínico, não controlado com 24 voluntários de ambos os sexos, que receberam ambulatorialmente $15 \mathrm{~mL}$ do xarope quatro vezes ao dia, durante 21 dias ininterruptos. Os voluntários foram incluídos no estudo somente quando considerados saudáveis após avaliação clínica, exame físico e exames laboratoriais que antecederam o estudo. $\mathrm{O}$ xarope Calmatoss ${ }^{\circledR}$ foi bem tolerado pelos 24 voluntários não apresentando eventos adversos graves. Os exames clínicos, eletrocardiográficos e laboratoriais efetuados antes, durante e após o ensaio não evidenciaram sinais de toxicidade nos diversos órgãos e sistemas avaliados, confirmando a segurança da preparação para utilização em ensaios de eficácia terapêutica.
\end{abstract}

Unitermos: Mikania glomerata, Grindelia robusta, Copaifera officinalis, Myroxylon toluifera, Nasturtium officinale, toxicologia, fitoterápico.

\begin{abstract}
Clinical safety evaluation of a phytomedicine containing Mikania glomerata, Grindelia robusta, Copaifera officinalis, Myroxylon toluifera, Nasturtium officinale, honey and propolis in healthy volunteers". Calmatoss ${ }^{\circledR}$ is a phytomedicine used in several respiratory tract pathologies treatment composed of seven medicinal plants such as Mikania glomerata, Grindelia robusta, Copaifera officinalis, Myroxylon toluifera, Nasturtium officinale, as well as honey and propolis. The present study investigated the chronic administration of $15 \mathrm{~mL}$ Calmatoss ${ }^{\circledR}$ syroup four times a day during 21 days for any toxic effect on healthy volunteers. The clinical trial consisted of an open study with 24 volunteers included in the study only when considered healthy after clinical evaluation, physical examination and laboratory tests, which preceded the study. The laboratory tests included: heamatologic, biochemical and sorologic analysis. This evaluation was repeated after the first, second and third week of treatment and at post-study seven days after the last administration. Calmatoss ${ }^{\circledR}$ was well tolerated by the volunteers. Variations in the laboratory were observed and all of these laboratory changes returned to normal levels during or after the study. In spite of these variations none of the volunteers had their values out of the established normality limits for each parameter. In conclusion, clinical, electrocardiographic and laboratory tests did not show any evidence of toxic signals in the various organs and systems studied.
\end{abstract}

Keywords: Mikania glomerata, Grindelia robusta, Copaifera officinalis, Myroxylon toluifera, Nasturtium officinale, toxicology, phytotherapic.

\section{INTRODUÇÃO}

O fitoterápico Calmatoss ${ }^{\circledR}$ é um xarope utilizado comoantitussígeno, expectorante, antissépticorespiratório e broncodilatador. É composto por tinturas Mikania glomerata Spreg. (Asteraceae), Grindelia robusta Nutt.
(Asteraceae), Copaifera officinalis (Jacq.) L. (Fabaceae), Myroxylon toluifera L. Harms. (Fabaceae), Nasturtium officinal R. Br.(Brassicaceae), além de própolis e mel. Seu registro e comercialização é baseado no uso popular de tais substâncias em afecções do trato respiratório.

A Mikania glomerata conhecida popularmente 
como guaco tem informações etnofarmacológicas que lhe atribuem as propriedades tônica, depurativa, antipirética, estimulante do apetite, no tratamento da gripe (Coimbra, 1942, Cortez et al., 1999; Pereira et al., 2004), além de ser indicado para inflamações da garganta, utilizando-se as folhas cozidas para gargarejo (Matos, 2000). Algumas ações sobre as vias respiratórias foram comprovadas experimentalmente e são justificadas pelos seus efeitos antialérgicos (Fierro et al., 1999) broncodilatadores (Soares et al., 2002a), antimicrobianos (Barbosa et al., 1994, Oliveira et al., 2000, Holetz et al., 2002; Duarte et al., 2004; Do Amaral et al., 2003) e antiedematogênicos (Oliveira et al., 1985, Leite et al., 1992). Outras atividades descritas na literatura para M. glomerata são atividade antiinflamatória (Falcão et al., 2005) e antidiarréica (Salgado et al., 2005).

A grindélia ou Grindelia robusta é uma planta largamente utilizada nas enfermidades da pele, trato respiratório e digestivo (Alonso, 1998). A parte da planta empregada são as flores, ricas em grindelina e mucilagens que proporcionam efeito expectorante que, somado com ações bactericidas, antiinflamatórias e espasmolíticas dos ácidos fenólicos e flavonóides, geram bons resultados em casos de bronquites, laringites e asma (Cañigueral; Vila, 1998, Mills; Bone, 2000).

De acordo com Alonso (1998) o Bálsamo de Tolú, Myroxylon toluifera ou Toluifera balsamum é originário da América do Sul (Venezuela, Colômbia e Peru). A oleoresina extraída do tronco mediante cortes em "V" é rica em substâncias com propriedades antisépticas expectorantes (Boyd, 1954, Melo; Melo 2005), espasmolíticas, cicatrizantes e antiparasitárias (Alonso, 1998). É empregado topicamente no tratamento de sarnas, feridas e úlceras dérmicas (Cañigueral; Vila, 1998) e na

Tabela 1. Eventos adversos relatados pelos voluntários $(n=24)$ durante o período de administração do xarope Calmatoss ${ }^{\circledR}$.

\begin{tabular}{l|c|c}
\hline Evento Adverso & $\mathrm{N}^{0}$ de observações & Freqüência (\%) \\
\hline Cefaléia & 4 & 16,6 \\
Dismenorréia & 2 & 8,3 \\
Sonolência & 2 & 8,3 \\
Gripe & 1 & 4,16 \\
Astenia & 1 & 4,16 \\
Faringite & 1 & 4,16 \\
Tosse & 1 & 4,16 \\
\hline
\end{tabular}

Tabela 2. Resultados dos exames laboratoriais obtidos dos 24 voluntários saudáveis durante o período de tratamento por via oral na dose $60 \mathrm{~mL}$ por dia do xarope Calmatoss ${ }^{\circledR}$, comparados aos valores obtidos antes e após a administração do fitoterápico.

\begin{tabular}{l|cccc}
\hline \multirow{2}{*}{ Parâmetros Laboratoriais } & \multicolumn{2}{|c}{ Mulheres } & \multicolumn{2}{c}{ Homens } \\
\cline { 2 - 5 } & Antes & Depois & Antes & Depois \\
\cline { 2 - 5 } Albumina (g/dL) & $4,09 \pm 0,2$ & $4,09 \pm 0,2$ & $4,3 \pm 0,2$ & $4,3 \pm 0,2$ \\
ALT (U/L) & $22,4 \pm 13,5$ & $18,5 \pm 10,7$ & $20,9 \pm 11,4$ & $18,6 \pm 13,2$ \\
AST (U/L) & $23,67 \pm 8,63$ & $20,8 \pm 5,4$ & $22,3 \pm 6,5$ & $17,4 \pm 4,5$ \\
Bilirrubina (mg/dL) & $0,61 \pm 0,16$ & $0,47 \pm 0,17$ & $0,8 \pm 0,3$ & $0,6 \pm 0,3$ \\
Creatinina (mg/dL) & $0,54 \pm 0,1$ & $0,63 \pm 0,1$ & $0,80 \pm 0,1$ & $0,83 \pm 0,1$ \\
Fosfatase alcalina (U/L) & $140,9 \pm 44,9$ & $144,3 \pm 50,0$ & $198,8 \pm 48,6$ & $193,8 \pm 64,4$ \\
Gamma GT (U/L) & $15,8 \pm 5,5$ & $16,8 \pm 6,0$ & $26,0 \pm 16,7$ & $15,6 \pm 20,7$ \\
Glicemia (mg/dL) & $82,9 \pm 8,5$ & $82,3 \pm 10,8$ & $84,9 \pm 6,2$ & $87,3 \pm 8,1$ \\
Hematócrito (\%) & $40,61 \pm 3,1$ & $40,22 \pm 2,9$ & $42,83 \pm 2,9$ & $42,6 \pm 3,9$ \\
Hemoglobina (g/dL) & $13,8 \pm 1,3$ & $13,4 \pm 1,2$ & $14,7 \pm 1,0$ & $14,3 \pm 1,1$ \\
Leucócitos (cels/mm $\left.{ }^{3}\right)$ & $7,1 \pm 1,3$ & $7,2 \pm 0,8$ & $6,5 \pm 1,5$ & $6,2 \pm 1,4$ \\
Plaquetas (cels/mm $\left.{ }^{3}\right)$ & $272,3 \pm 6,0$ & $299,6 \pm 55,5$ & $242,7 \pm 28,7$ & $237,4 \pm 32,6$ \\
Potássio (Mmol/L) & $4,1 \pm 0,3$ & $4,2 \pm 0,2$ & $4,3 \pm 0,4$ & $4,2 \pm 0,2$ \\
Proteína total & $7,4 \pm 0,4$ & $7,3 \pm 0,3$ & $7,4 \pm 0,3$ & $7,2 \pm 0,3$ \\
Sódio (Mmol/L) & $138,8 \pm 2,6$ & $138,8 \pm 1,0$ & $140,4 \pm 1,9$ & $138,8 \pm 3,2$ \\
TP & $1,1 \pm 0,1$ & $1,2 \pm 0,1$ & $1,2 \pm 0,1$ & $1,1 \pm 0,1$ \\
TPTA (\%) & $1,0 \pm 0,1$ & $1,0 \pm 0,1$ & $1,0 \pm 0,1$ & $1,0 \pm 0,1$ \\
Triglicérides (mg/dL) & $73,5 \pm 27,6$ & $74,0 \pm 31,4$ & $96,1 \pm 45,7$ & $96,6 \pm 43,8$ \\
Uréia (mg/dL) & $21,8 \pm 3,6$ & $26,9 \pm 4,8$ & $28,8 \pm 6,2$ & $29,1 \pm 5,7$ \\
\hline
\end{tabular}


indústria como corretor organoléptico (Cañigueral; Vila, 1998, Scardamaglia et al., 2003).

O agrião ou Nasturtium officinale é uma planta largamente utilizada na medicina popular brasileira (Matos, 2000) empregando-se as partes aéreas no tratamento de bronquites, gripes, faringites e laringites (Cañigueral; Vila, 1998). É comprovadamente seguro em animais em doses terapêuticas (Zaupa et al., 2002) e apresenta ação inibitória no metabolismo oxidativo de algumas substâncias como acetaminofeno (Chen et al., 1996) e cumarina (Murphy, 2001) e como antidiabética (Barbosa-Filho et al., 2005).

A copaíba ou Copaifera officinalis L. é uma árvore frondosa pertencente a família das Leguminosas. A parte utilizada é a resina, extraída mediante cortes feitos no caule (Alonso, 1998). As ações farmacológicas estão relacionadas com ações antimicrobianas (Arrhenius; Langenheim, 1983) e antiinflamatórias, principalmente do sistema respiratório (Basile et al., 1988; Handa et al., 1992) e urinário (Paiva et al., 2003).

O própolis é uma das poucas substâncias naturais que se mantém popular ao longo dos anos (Castaldo; Capasso, 2002) e encontra adesão em algumas redes públicas de saúde como fitoterápico (Ogava et al., 2003). Os componentes químicos farmacologicamente ativos são os flavonóides, os ácidos fenólicos e seus ésteres (Borrelli et al., 2002, Khayyal et al., 1993) que possuem efeitos sobre bactérias (Koo et al., 2000), vírus (Vynograd et al., 2000), fungos (Tosi et al., 1996), ações antiinflamatórias (Rossi et al., 2002) e imunomoduladoras (Dimov et al., 1992) além de exercer possíveis efeitos sobre a redução da pressão arterial (Ricchuto, 1994) e dos níveis de colesterol (Claus et al., 2000).

Apesar do uso dessas substâncias ser difundido entre a população brasileira e estarem descritas na Farmacopéia Brasileira (Brandão et al., 2006) nos consta que há um único registro clínico de eficácia e segurança com uma formulação semelhante a nossa, composto pela associação das plantas Mikania glomerata, Mentha piperita, Eucalyptus globulus e Copaifera multifuga, incorporadas ao mel e à própolis, denominado xarope Saratose ${ }^{\circledR}$ (Tavares et al., 2006). De acordo com o Food and Drug Administration (FDA) e a Agência Nacional de Vigilância Sanitária (ANVISA), a primeira etapa de experimentação de medicamentos em seres humanos, se inicia por questões éticas, pela avaliação da segurança em uma amostra de voluntários saudáveis para depois verificar a sua eficácia. O objetivo desse trabalho foi avaliar a segurança clínica da associação de Mikania glomerata, Grindelia robusta, Copaifera officinalis, Myroxylon toluifera, Nasturtium officinale, própolis e mel em voluntários saudáveis.

\section{MATERIAL E MÉTODOS}

\section{Desenho do experimento}

O estudo realizado consistiu de um ensaio clínico aberto não controlado, com 24 voluntários saudáveis, com idade de 18 a 50 anos.

\section{Seleção dos voluntários}

Os voluntários foram previamente submetidos ao exame físico, história clínica, ECG, e exames laboratoriais complementares. Participaram do ensaio homens e mulheres saudáveis, $\beta$-HCG negativo, de 18 a 50 anos de idade, com índice de massa corpórea (IMC) de 18 a $30 \mathrm{~kg} / \mathrm{m}^{2}$. Os voluntários foram incluídos no estudo somente quando considerados saudáveis após avaliação das funções hepática, renal, cárdio-respiratória , através da história clínica, exame físico e os exames laboratoriais que antecederam o estudo (Moraes et al., 2004). Essa mesma avaliação foi repetida após a primeira, segunda, terceira semana de tratamento e sete dias após o término estudo.

Constaram dos exames laboratoriais: a análise hematológica através da contagem da hemoglobina, hematócrito, contagem total e diferencial de leucócitos e contagem de plaquetas; análise bioquímica através do sódio, potássio, creatinina, bilirrubina, proteínas totais, albumina, glicemia, fosfatase alcalina, AST, ALT, colesterol total, triglicérides, ácido úrico, $\gamma \mathrm{GT}$ e sumário de urina. A análise sorológica para hepatite $\mathrm{B}$, hepatite $\mathrm{C}$, HIV e $\beta \mathrm{HCG}$ foram realizadas somente no pré-estudo.

Foram excluídos do estudo voluntários com história de doença hepática, renal, cardíaca ou epiléptica. Além daqueles que, antes do estudo, fizeram uso de medicação regular por até 2 semanas, ingestão de droga experimental por até 3 meses, doação ou perda de 450 $\mathrm{mL}$ de sangue ou mais até 3 meses antes do estudo, abuso de álcool ou drogas e internação hospitalar até 8 semanas seguindo protocolo descrito por Soares et al. (2003) para estudo de toxicologia clínica.

\section{Administração do fitoterápico}

Os voluntários receberam $15 \mathrm{~mL}$ do xarope Calmatoss ${ }^{\circledR}$ (lote 642022, fabricado pela Herbarium Laboratório Botânico Ltda., Brasil), por via oral 4x/dia, seguido de $200 \mathrm{~mL}$ de água, esse tratamento foi repetido durante 21 dias.

De acordo com o protocolo experimental, os voluntários deveriam comparecer diariamente a Unidade de Farmacologia Clínica da UFC para administrações do fitoterápico às 7,11 e 17 hs, momento em que verificações de sinais vitais (pressão arterial e pulso radial) também eram efetuados. A última dose do dia ( $22 \mathrm{hs}$ ) era feita pelo próprio paciente em domicílio. Após a administração das 17 hs os voluntários recebiam medicação para a próxima dose e orientação de como fazê-la.

Os voluntários permaneceram em suas atividades rotineiras e mantiveram as suas dietas gerais normais. Também foram orientados a relatar qualquer

\begin{tabular}{r|c} 
Rev. Bras. Farmacogn. & 449 \\
Braz J. Pharmacogn. & \\
16(4):out/dez. 2006 &
\end{tabular}


evento adverso, a hora de ocorrência e uso de medicação concomitante. Os eventos adversos foram avaliados de acordo com a causalidade pela classificação de Karch e Lasagna (1975).

\section{Aspectos éticos}

$\mathrm{O}$ projeto de pesquisa, com o protocolo e o termo de consentimento foram submetidos e aprovados pelo Comitê de Ética em Pesquisa da UFC, credenciado pelo Ministério da Saúde.

Todos os voluntários foram esclarecidos sobre a natureza e os objetivos do estudo e aqueles que concordaram em participar deram seu consentimento formal e escrito através da assinatura do termo de consentimento

\section{Análise estatística}

Os resultados dos exames laboratoriais foram avaliados individualmente verificando se esses se encontravam dentro das faixas de referência. A ocorrência de variações significativas em relação ao período sem medicação (controle) foi comparada através de análise de variância (ANOVA) seguida do teste de Dunnet $(\mathrm{p}<0,05)$ e a correlação de Spearman. Os gráficos foram feitos utilizando o software Graph Pad Prism Versão 2.01 e as análises estatísticas no programa SPSS Versão 10.0.

\section{RESULTADOS}

Foram selecionados 24 voluntários saudáveis ( 12 homens e 12 mulheres) com idade média de 27,08 \pm 7,6 anos. Os voluntários selecionados apresentavam IMC de $22,57 \pm 3,18 \mathrm{~kg} / \mathrm{m}^{2}$ não havendo diferenças significativas no pós-estudo $(\mathrm{p}=0,42)$.

A formulação foi bem tolerada por todos os voluntários, entretanto alguns eventos adversos foram relatados. Estes, apesar de ocorrerem durante o período de estudo não tiveram relação com a administração do Calmatoss ${ }^{\circledR}$ como evidenciado pela avaliação clínica. O evento adverso mais descrito foi cefaléia, seguida de dismenorréia, gripe, astenia, faringite e tosse (Tabela 1). $\mathrm{O}$ uso de medicação adicional somente foi necessário nos voluntários que apresentaram episódios de cefaléia onde se utilizou Neosaldina ${ }^{\circledR}$, Paracetamol e Cefaliv ${ }^{\circledR}$ e na dismenorréia das voluntárias que relataram uso de Paracetamol, Meloxicam e Cetoprofeno para controle da dor (cada voluntário fez uso de uma única medicação em dose única para o tratamento dos eventos adversos citados).

Nenhum dos eventos descritos foi considerado relacionado com a administração do fitoterápico e foram observados em voluntários que desenvolveram infecções virais ou bacterianas do trato respiratório no período de estudo, ou em voluntária durante período menstrual retirando possibilidade de associação com o tratamento empregado. Medicações para tratamento de reações adversas foram permitidas uma vez que os eventos eram leves e não exigiam a retirada do voluntário do estudo nem a interrupção do tratamento como o xarope Calmatoss ${ }^{\circledR}$, além do fato que uma re-exposição ao fitoterápico em estudo poderia favorecer o reaparecimento de eventos adversos e confirmar ou não sua associação ao tratamento.

Dois voluntários referiram sonolência de leve intensidade durante algumas semanas do estudo, esse evento adverso foi considerado como de causa duvidosa usando a classificação de reações adversas de acordo com a causalidade de Karch e Lasagna (1975).

Os exames laboratoriais, eletrocardiograma e exames médicos encontravam-se em geral, dentro dos limites de normalidade durante cada semana de administração (Figuras 1-3). Não se observaram variações estatisticamente significantes quando se comparou a avaliação durante a administração com o período basal dos voluntários $(\mathrm{p}>0,05)$ como observado na tabela 2 .

\section{DISCUSSÃO}

Embora já tenham sido realizados estudos préclínicos com as plantas presentes na formulação com o objetivo de mostrar suas atividades isoladas no trato respiratório provando o uso popular dessas plantas, um único estudo clínico foi realizado recentemente pelo nosso grupo, com uma associação semelhante (Tavares et al., 2006), para garantir a segurança dessas preparações em seres humanos.

Ensaios clínicos que objetivam avaliar segurança de fármacos são classificados como estudos de fase I, sendo realizados em uma amostra pequena (Moraes et al., 2004), que pode variar de 20 a 80 voluntários saudáveis ou pacientes (Pocock, 1987) ou 8 a 12 em estudos de doses repetidas realizados num período mínimo de 14 dias (Mant; Spector, 1994). Esses dados reforçam o protocolo adotado de 21 dias de administração em 24 voluntários saudáveis.

As doses empregadas em estudos de fase I são administradas de forma crescente até atingir as doses terapêuticas (Mant; Spector, 1994). Em geral inicia-se com uma dose correspondente de 1 a $2 \%$ da dose que induz os primeiros sintomas ou sinais de toxicidade nas espécies animais mais sensíves, posteriormente todo o processo é repetido em doses superiores, não existindo padrões para essa elevação (Oliveira, 2002). Neste caso, por se tratar de um fitoterápico de uso tradicional já utilizado pela população, resolvemos adotar a dose máxima diária indicada pelo fabricante da formulação que correspondia a $60 \mathrm{ml}$ por dia.

Apesar de conhecidas algumas reações adversas da copaíba como náusea, vômitos, cólicas com diarréia especialmente em doses altas (Souza-Júnior et al., 2000), ou das reações alérgicas do própolis sobre pele e membranas (Castaldo; Capasso, 2002) não há evidências 

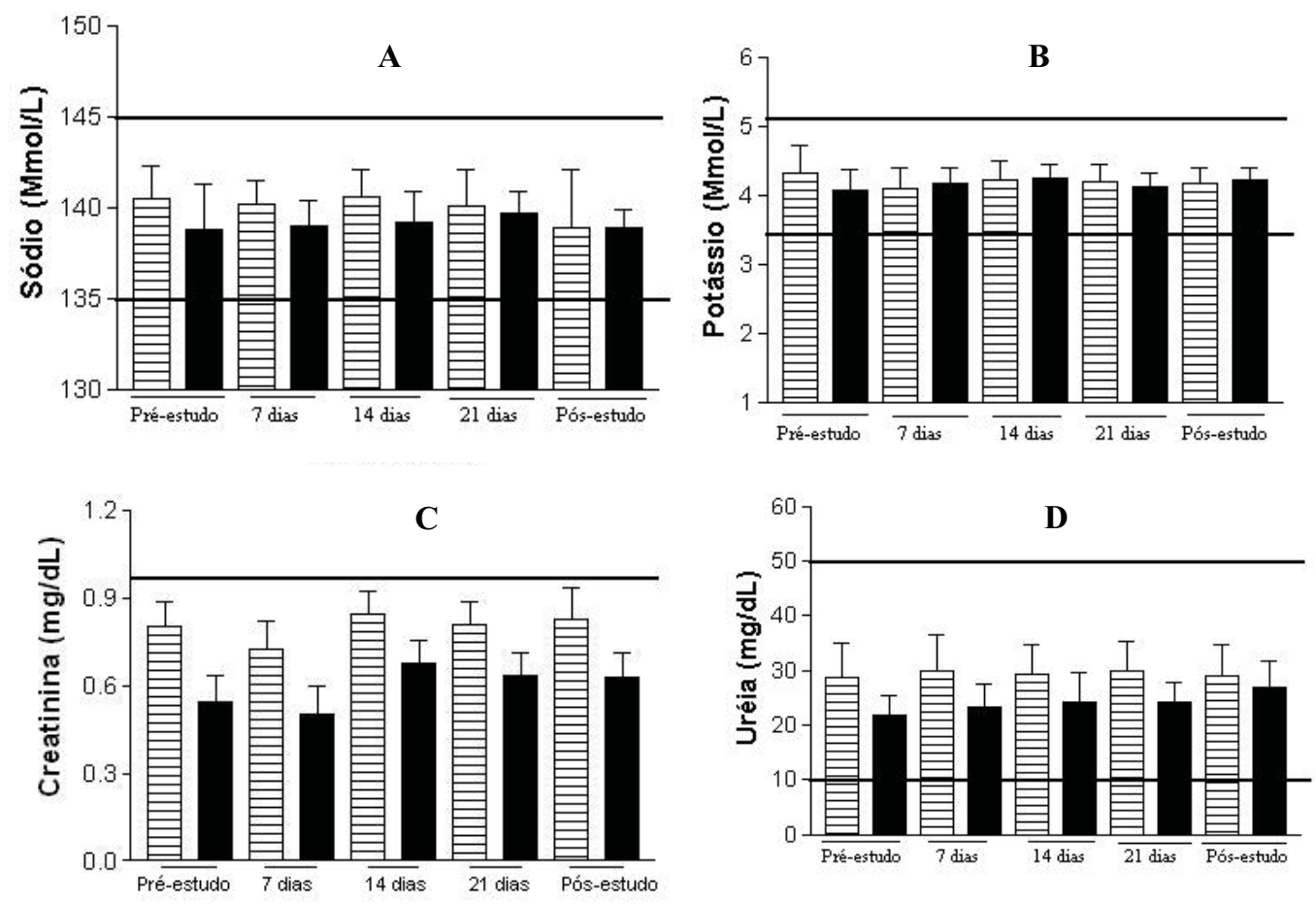

Figura 1. Comparação dos parâmetros laboratoriais da função renal (média \pm DP) de cada semana de tratamento em relação ao período sem medicação (pré-estudo) em voluntários saudáveis do sexo masculino e feminino referência $(-)$ para ambos os sexos.
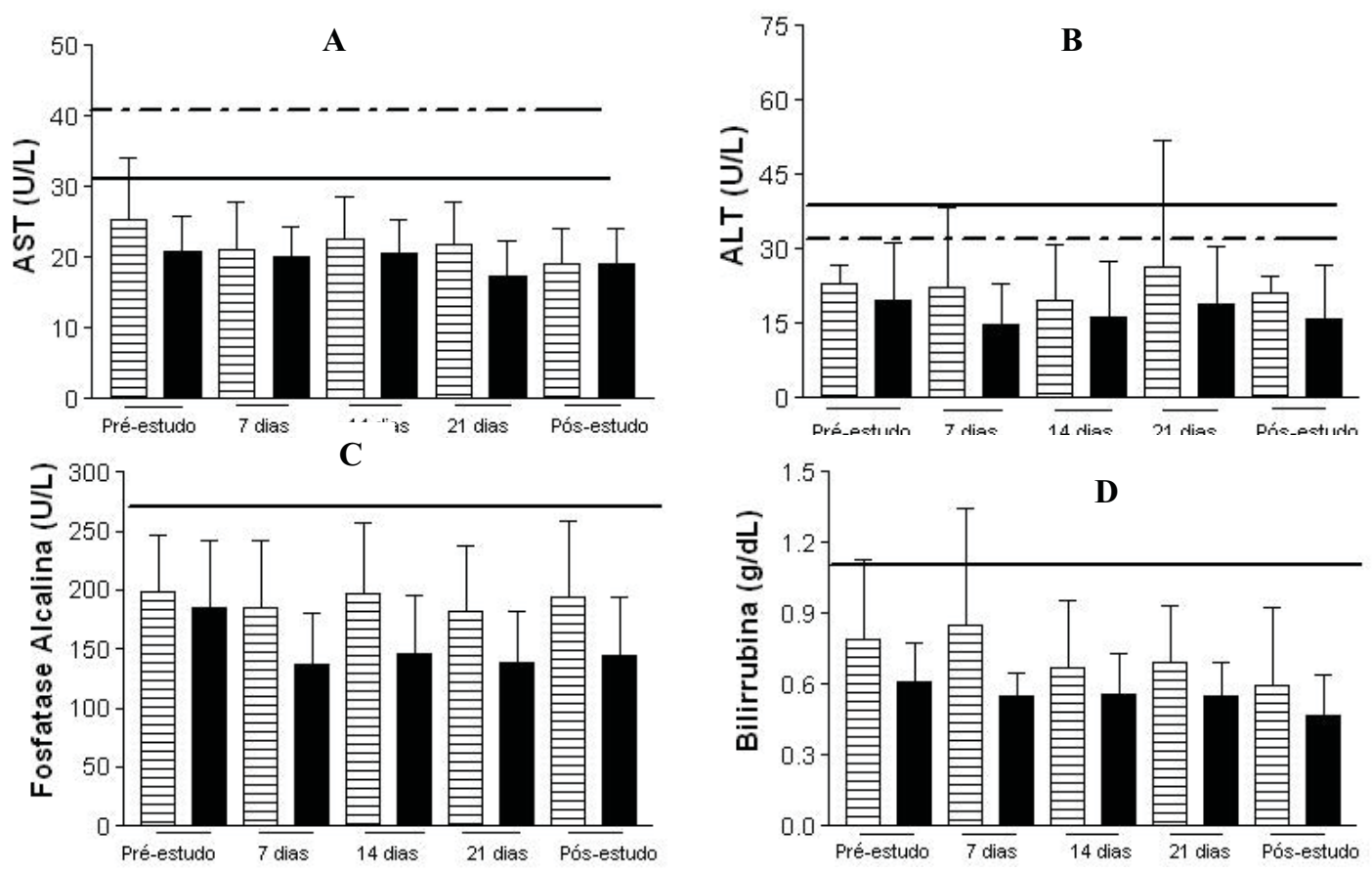

Figura 2. Função hepática (média \pm DP) de cada semana de tratamento em relação ao período préestudo em voluntários saudáveis do sexo masculino e feminino apresentam duas linhas (A e B) possuem valores de referência que diferem com o sexo para mulheres (一) e homens (----). Para os gráficos $\mathrm{C}$ e $\mathrm{D}$ a linha (-) representa valores de referência para ambos os sexos.

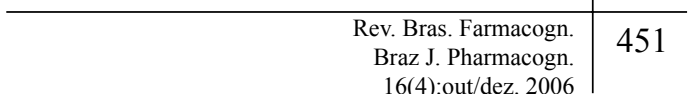


A

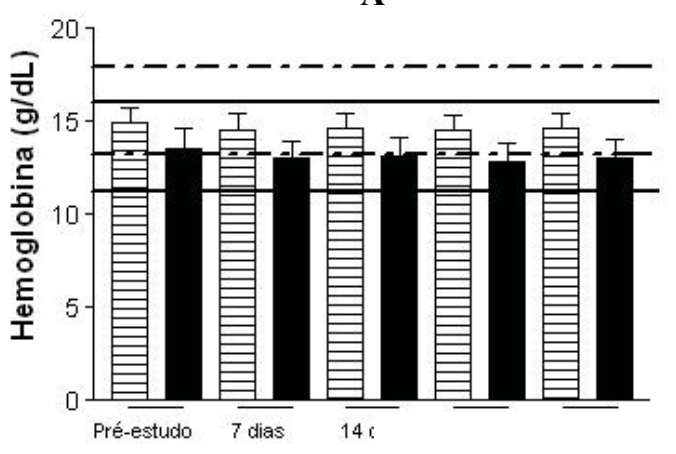

B

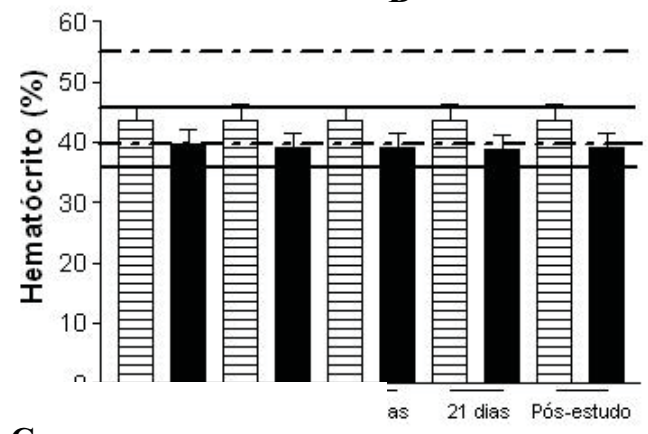

C

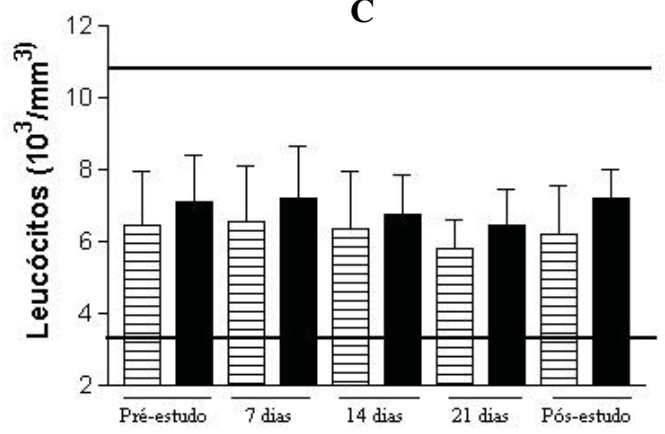

Figura 3. Função hematológica (média \pm DP) de cada semana de tratamento em relação ao período pré-estudo em voluntários saudáveis do sexo masculino $\square$ e feminino apresentam duas linhas (A e B) possuem valores de referência que diferem com o sexo, ( - ) para mulheres e homens (----). Para o gráfico $\mathrm{C}$, a linha contínua $(-)$ representa valores de referência para ambos os sexos.

de reações adversas relacionadas com a administração do fitoterápico durante os 21 dias de administração.

A análise dos exames laboratoriais comparados ao período antes do tratamento não mostrou significância estatística que apontasse toxicidade nos diversos órgãos e sistemas avaliados. As variações dos exames laboratoriais observadas nas avaliações semanais apresentaram-se dentro da faixa de normalidade para cada parâmetro, tampouco apresentaram sinais de toxicidade de acordo com a Organização Mundial de Saúde (Soares et al., 2002b, Who, 2003).

A presença de substâncias com conhecida ação sobre inibição da coagulação sanguínea (Suttie, 1987) como é o caso dos compostos cumarínicos em plantas como a Mikania glomerata (Matos, 2000), não foi capaz de gerar nenhuma alteração clinicamente significante durante o período de tratamento nessa função como observado nos valores do TP e TPTA. Esses parâmetros avaliam as vias da coagulação extrínsecas e intrínsecas respectivamente, e a ausência de alterações nesses valores, provavelmente se deve as baixas concentrações desses compostos cumarínicos na formulação.

O ensaio clínico realizado caracterizou um estudo fase I, onde uma droga é administrada pela primeira vez em seres humanos para determinação de sua segurança (Mant; Spector, 1994). Essa etapa clínica mesmo se mostrando segura não garante ausência de reações adversas das formulações testadas, especialmente aquelas de baixa incidência que somente seriam observadas após utilização por centenas ou milhares de pacientes. A detecção de reações adversas deverão continuar sendo monitoradas no período pós-comercialização pelos programas de farmacovilância (Ferreira, 2002, Moraes; Moraes 2000).

Conclui-se, portanto, que administração do xarope Calmatoss ${ }^{\circledR}$ quatro vezes ao dia ( $15 \mathrm{~mL}$ por dose) durante 21 dias mostrou-se segura, pois não induziu aparecimento de nenhuma reação adversa severa nos voluntários saudáveis. Esse fato permite a realização de novos estudos para avaliação de suas indicações terapêuticas e ajuste posológico.

\section{REFERÊNCIAS}

Alonso JR 1998. Tratado de fitomedicina. Buenos Aires: Isis Ediciones SRL.

Arrhenius SP, Langenheim JH 1983. Inhibitory effects of Hymenaea and Copaifera leaf resins on the leaf fungus Pestalotia subcuticularis. Biochem Syst Ecol 11: 361-366.

Barbosa AD, Ferreira RCV, Valente PHM 1994. Atividade antimicrobiana de extratos fluidos de plantas medicinais brasileiras. Lecta-USF 12: 153-163.

Barbosa-Filho JM, Vasconcelos THC, Alencar AA, Batista LM, Oliveira RAG, Guedes DN, Falcão HS, Moura 
MD, Diniz MFFM, Modesto-Filho J 2005. Plants and their active constituents from South, Central, and North America with hypoglycemic activity. Rev Bras Farmacogn 15: 392-413.

Basile A, Sertie J, Freitas P, Zanini A 1988. Anti-inflammatory activity of oleoresin from brazilian copaifera. $J$ Ethnopharmacol 22: 101-109.

Borrelli F, Maffia P, Pinto L, Ianaro A, Russo A, Capasso F, Ialenti A 2002. Phytochemical compounds involved in the anti-inflamatory effect of própolis extract. Fitoterapia 73: S53-S63.

Boyd EM. Expectorants and resoiratory tract fluid 1954. Pharmacol Rev 6: 521-542.

Brandão MGL, Cosenza GP, Moreira RA, Monte-Mor RLM 2006. Medicinal plants and other botanical products from the Brazilian Official Pharmacopoeia. Rev Bras Farmacogn 16: 408-420.

Cañigueral S, Vila R 1998. Fitoterapia, vademecum de prescripción. 3.ed. Barcelona: Masson.

Castaldo S, Capasso F 2002. Propolis, an old remedy used in modern medicine. Fitoterapia 73: S1-S6.

Chen L, Mohr SN, Yang CS 1996. Decreased of plasma and urinary oxidative metabolites of acetaminophen after consumption of watercress by human volunteers. Clin Pharmacol Ther 60: 651-660.

Claus R, Kinscherf R, Gehrke C, Bonaterra G, Basnet P, Metz J, Deigner HP 2000. Antiapoptotic effects of propolis extract and propol on human macrophages exposed to minimally modified low density lipoprotein. Arzneimittel-Forsch 50: 373-379.

Coimbra R 1942. Notas de fitoterapia. Rio de Janeiro: Ed. L.C.S.A.

Cortez LER, Jacomossi E, Cortez DAG 1999. Levantamento de plantas medicinais usadas na medicina popular de Umuarama, PR. Arquivos de Ciências da Saúde UNIPAR 3: 97-104.

Dimov V, Ivanovska N, Bankova V, Popov S 1992. Immunomodulatory action on propolis: IV. Profilactic activity against gram-negative infections and adjuvant effect of the wather-soluble derivative. Vaccine 10: 817-823.

Duarte MCT, Figueira GM, Pereira B, Magalhães PM, Delarmelina C 2004. Atividade antimicrobina de extratos hidroalcoólicos de espécies da coleção de plantas medicinais CPQBA/UNICAMP. Rev Bras Farmacogn 14(Supl. 1): 6-8.

Falcão HS, Lima IO, Santos VL, Dantas HF, Diniz MFFM, Barbosa-Filho JM, Batista LM 2005. Review of the plants with anti-inflammatory activity studied in Brazil. Rev Bras Farmacogn 15: 381-391.

Ferreira EI 2002. Como nascem e se desenvolvem os novos medicamentos. In: Silva P. Farmacologia. 6.ed. Rio de Janeiro: Guanabara Koogan.. p.200-208.

Fierro IM, Da Silva AC, Lopes CS, Moura RS, Barja-Fidalgo C 1999. Studies on the anti-allergic activity of Mikania glomerata. J Etnopharmacol 66: 19-24.

Handa S, Chawla A, Sharma A 1992. Plants with antiinflamatory activity. Fitoterapia 68: 3-31.

Holetz FB, Pessini GL, Sanches NR, Cortez DA, Nakamura CV, Filho BP 2002. Screening of some plants used in brazilian folk medicine for the treatment of infectous diseases. Mem I Oswaldo Cruz 97: 1027-1031.

Karch FE, Lasagna L 1975. Adverse drug reaction. A critical review. JAMA 234: 1236-1241.

Khayyal MT, El-Ghazaly MA, El-KhatibAS 1993. Mechanisms involved in the antiinflammatory effect of propolis extract. Drugs Exp Clin Res 19: 197-203.

Koo H, Gomes BP, Rosalen PL, Ambrosano GM, Park YK, Cury JA 2000. In vitro antimicrobial activity of própolis and Arnica montana against oral pathogens. Arch Oral Biol 45: 141-148.

Leite MGR, Silva MAM, Lino CS, Viana GSB, Matos FJA 1992. Atividade broncodilatadora em Mikania glomerata, Justicia pectoralis e Torresea cearensis. Resumos do XII Simpósio de Plantas Medicinais do Brasil, Curitiba-PR, p.021.

Mant TGK, Spector RG 1994. Phase 1 studies and pharmacokinetics. In: Lloyd J., Raven A. Handbook of clinical research 2nd. ed. New York: Churchill Livingstone.

Matos FJA 2000. Plantas medicinais: Guia de seleção e emprego de plantas usadas em fitoterapia no nordeste do Brasil. 2ed. Fortaleza: Imprensa Universitária - UFC.

Melo FB, Melo JRB 2005. Efeitos antitussígenos e expectorantes de dois fitoterápicos comercializados no mercado brasileiro. Acta Scientiae Veterinariae 33: 161-167.

Mills S, Bone K 2000. Principles and pratices of phytotherapy. 3ed. London: Churchill Livingstone.

Moraes MEA, Moraes MO 2000. Ensaios clínicos de medicamentos no Brasil. Fármacos \& Medicamentos 6: 36-40.

Moraes MO, Bezerra FAF, Lotufo LC, Pessoa C, Moraes MEA 2004. Avaliação clínica da eficácia e segurança de fitoterápicos no Brasil. Arq Bras Fitomed Cient 1: 30-39.

Murphy SE 2001. Consumption of watercress fal to alter coumarin metabolism in humans. Drug Metab Disp 29: 786-787.

Ogava SEM, Pinto MTC, Kikuchi T, Menegueti VAF, Martins DBC, Coelho SAD, Marques MJNJ, Virmond JCS, Monteschio P, D'Aquino M, Marques LC 2003. Implantação do programa de fitoterapia "Verde Vida" na secretaria de saúde de Maringá (2000-2003). Rev Bras Farmacogn 13(Supl. 1): 58-62.

Oliveira F, Oga S, Akisue G, Akisue MK 1985. Parâmetros físicos e químicos e efeito antiedema dos extratos fluidos de guaco (Mikania glomerata Sprengel) e guaco de mato (Mikania laevigata Schultz Bip. Ex Baker). An Farm Quim 25: 50-54.

Oliveira F, Rodrigues RFO, Bastos DHM, Pereira MH 2000. Caracterização morfohistológica e verificação da atividade microbiológica da espécie vegetal Mikania cordifolia (Lf) Willd. Lecta-USF 18: 33-63.

Oliveira GG 2002. Os ensaios clínicos. In: Silva, P. Farmacologia. 6.ed. Rio de Janeiro: Guanabara Koogan. p.146-55.

Paiva LAF, Gurgel LA, Silva RM, Tomé AR, Gramosa NV, Silveira ER, Santos FA, Rao VSN 2003. Antiinflammatory effect of kaurenoic acid, a diterpene from Copaifera langsdorffii on acetic acid-induced colitis in rats. Vasc Pharmacol 39: 303-307.

Pereira RC, Oliveira MTR, Lemos GCS 2004. Plantas utilizadas como medicinais no município de Campos de Goytacazes - RJ. Rev Bras Farmacogn 14 (Supl. 
1): 37-40.

Pocock SJ 1987. Clinical trials: A Practical approach. 5ed. London: John Wiley \& Sons Ltda.

Ricchiuto GM 1994. Le nuove frontiere della propoli. Verona: GMR Editore.

Rossi A, Longo R, Russo A, Borrelli F, Sautebin L 2002. The role of the phenethyl ester of caffeic acid (CAPE) in the inhibition of rat lung cyclooxygenase. Fitoterapia 73(Suppl 1):S30-S37.

Salgado HRN, Roncari AFF, Moreira RRD 2005. Antidiarrhoeal effects of Mikania glomerata Spreng. (Asteraceae) leaf extract in mice. Rev Bras Farmacogn 15: 205208.

Scardamaglia L, Nixon R, Fewings J 2003. Compound tinture of benzoin: a common contact allergen?. Aust $J$ Dermatol 44: 180-184.

Soares MR, Costa SS, Jansen JM, Silva CA, Lopes CS, Bernardo-Filho M, Silva VN, Criddle DN, Potela BN, Rubench LM, Araújo RG 2002a. Brochodilator activity of Mycania glomerata Sprengel on human bronchi and guinea-pig trachea. J Pharm Pharmacol 54: 249-256.

Soares JLMF, Pasqualotto AC, Rosa DD, Leite VRS 2002b. Métodos diagnósticos. Porto Alegre: Artmed.

Soares AKA, Martins IL, Uchoa CRA, Bezerra FAF, Moraes MO, Moraes MEA 2003. Clinical toxicology study of the phytomedicine Tamarine $\mathbb{R}$ in health volunteers. Arq Bras Fitomed Cient 1: 90-95.

Souza-Júnior OG, Damous SHB, Lamarão LG 2000. Revisão crítica do uso médico do óleo de copaíba. Rev Para Med 14: 71-76.

Suttie JW 1987. The biochemical basis of wafarin therapy. $A d v$ Exp Med Biol 214: 13-16.

Tavares JP, Martins IL, Vieira AS, Lima FAV, Bezerra FAF, Moraes MO, Moraes MEA 2006. Estudo de toxicologia clínica de um fitoterápico a base de associações de plantas, mel e própolis. Rev Bras Farmacogn 16: 350356.

Tosi B, Donini A, Romagnoli C, Bruni A 1996. Antimicrobial activity of some commercial extracts of propolis prepared with different solvents. Phytother Res 10: 335-336.

Vynograd N, Vynograd I, Sosnowski Z 2000. A comparative multi-centre study of the efficacy of propolis, acyclovir and placebo in the treatment of genital herpes (HSV). Phytomedicine 7:1-6.

Who (World Health Organization) 2003. Toxicity criteria by grade. [http://www. accessdata.fda.gov/scripts/ onctools/whotox.cfm] Acesso em 15/07/2003.

Zaupa C, Carraschi L, Tsuzuki JK, Boeira R, Silva JC, Marques LC 2002. Estudo toxicológico pré clínico (agudo e sub-agudo) do produto Protovit Plus ${ }^{\circledR}$ em roedores. Acta Farm Bonaerense 21: 264-272. 